\title{
Size-dependent alignment of Fe nanoparticles upon deposition onto W(110)
}

\author{
A. Kleibert, ${ }^{1,2, *}$ A. Voitkans, ${ }^{1,3}$ and K.-H. Meiwes-Broer ${ }^{1}$ \\ ${ }^{1}$ Institute of Physics, University of Rostock, D-18051 Rostock, Germany \\ ${ }^{2}$ Swiss Light Source, Paul Scherrer Institute, CH-5232 Villigen, Switzerland \\ ${ }^{3}$ Institute of Solid State Physics, University of Latvia, LV-1063 Riga, Latvia
}

(Received 28 December 2009; published 26 February 2010)

\begin{abstract}
Using in situ electron diffraction we study the orientation of mass-selected iron nanoparticles upon deposition onto single crystalline $\mathrm{W}(110)$ at room temperature. It is found that particles with a diameter below about $4 \mathrm{~nm}$ and a kinetic energy $\leq 0.1$ electron volt per atom spontaneously align with respect to the substrate. Larger particles preferentially rest with their (001) and (110) facets parallel to the surface, but do not show further alignment. The data may hint at thermally activated dislocation motions upon the impact on the substrate which are responsible for the observed orientation below $4 \mathrm{~nm}$. By this uniformly oriented monodisperse nanostructures can be prepared on single-crystalline substrates.
\end{abstract}

DOI: 10.1103/PhysRevB.81.073412

Size, shape, structure, and orientation of supported nanostructures have crucial impact on their electronic, optical, catalytic, and magnetic properties. ${ }^{1-4}$ Thus, gaining control over these parameters is subject of intense research and a number of methods has been developed for the preparation of monodisperse particles with uniform properties. ${ }^{5-8}$ Among them the deposition of preformed clusters has attracted much attention in the past since it enables stabilization of nanostructures far from thermal equilibrium and virtually any material and particle-size combination. ${ }^{9,10}$ It was found that the mobility, shape, and a possible epitaxial relation to the substrate are governed by the kinetic energy, the adsorption energy, and the lattice mismatch between the clusters and the substrate. ${ }^{6,9}$ It also turned out that epitaxy between deposited particles and a single-crystalline substrate can be achieved at relatively high impact energies or upon thermal annealing. ${ }^{11,12}$ However, under these conditions the particle shape as well as the substrate surface may not be conserved. ${ }^{11,13}$ Spontaneous epitaxial alignment at room temperature and under soft-landing conditions has been reported for particular cases, ${ }^{14,15}$ but an experimental investigation of size-dependent alignment phenomena and the mechanisms involved is still lacking, and thus, is the aim of the present contribution.

Epitaxial alignment is particularly an important issue with magnetic nanostructures, since it may enable the formation of particle arrays with uniformly oriented magnetization axes as required, e.g., in magnetic storage devices. From a fundamental point of view one may wish to understand in how far interface-related phenomena known from epitaxially grown films (e.g., strain and interface-induced contributions to the magnetocrystalline anisotropy) are also present in deposited nanoparticles. ${ }^{16}$ An intensively studied model system for the relation between epitaxial growth and the resulting magnetic properties of nanostructures is given by $\mathrm{Fe}$ on $\mathrm{W}(110) .{ }^{17} \mathrm{Fe}$ nanoparticles studied here possess a bulklike body-centered cubic (bcc) crystal structure with (110) and (001) surface facets forming a truncated dodecahedron according to the Wulff theorem, ${ }^{18} \mathrm{cf}$. the sketch further below in Fig. 3(e). Depending on their orientation upon deposition the particles may therefore show a similar crystallographic relation as epitaxially grown bcc(110) Fe films on W(110), or, alternatively, novel nonepitaxial interfaces as, e.g., $\mathrm{Fe}(001) / \mathrm{W}(110)$
PACS number(s): 61.46.Df, 61.05.jh, 68.35.-p, 75.75.-c

with so far unknown properties, which are hardly accessible by other means.

In this Brief Report we show by means of in situ electron diffraction that $\mathrm{Fe}$ nanoparticles below a critical particle diameter between 2 and $4 \mathrm{~nm}$ spontaneously align on W(110) (without the need of thermal annealing). Larger particles are found to land preferentially on their surface facets but with random azimuthal orientation with respect to the substrate lattice. In these experiments, we particularly benefit from the enhanced surface sensitivity provided by the grazing incidence in reflection high-energy electron diffraction (RHEED). While the W(110) substrate shows a well-known RHEED pattern determined by the two-dimensional surface lattice, the deposited nanoparticles represent threedimensional structures and are thus probed in transmission. Therefore, randomly oriented particles give rise to a DebyeScherrer ring pattern analogously to conventional powder diffraction. In contrast, ordered particles show distinct diffraction spots occurring and vanishing when azimuthally rotating the sample. Thereby the RHEED pattern of the W(110) substrate serves as a reference and, thus, allows us to study, both, the relative orientation of the particles with respect to the substrate and also possible strain in the nanoparticles.

The room-temperature experiments are performed in an ultrahigh-vacuum chamber system combining a substrate preparation stage with a RHEED apparatus and an arc cluster ion source (ACIS) ${ }^{19}$ The base pressure is $<1 \times 10^{-10} \mathrm{mbar}$. In our setup the ACIS focuses a continuous beam of sizeselected nanoparticles onto a W(110) crystal being aligned in the RHEED chamber, cf. Fig. 1(a). This enables us to follow changes in the electron-diffraction pattern during particle deposition. The RHEED system consists of a phosphorus screen [represented by a typical diffraction pattern of W(110) in the figure] and an electron gun. The electron energy is set to $25 \mathrm{keV}$. The azimuthal orientation of the sample can be varied by any angle $\phi$ between the W[110] axis and the electron beam. In the present experiments the angle of incidence ranges between $0.5^{\circ}$ and $2.5^{\circ}$. The actual sample orientation is obtained from analyzing the full W(110) RHEED pattern by means of Ewald sphere construction. The cluster source provides mass-filtered, pure metallic clusters with the diameter $D$ tunable in the range from 2 to $25 \mathrm{~nm} \cdot{ }^{20,21}$ The particle beam shows a narrow size distribution with a 


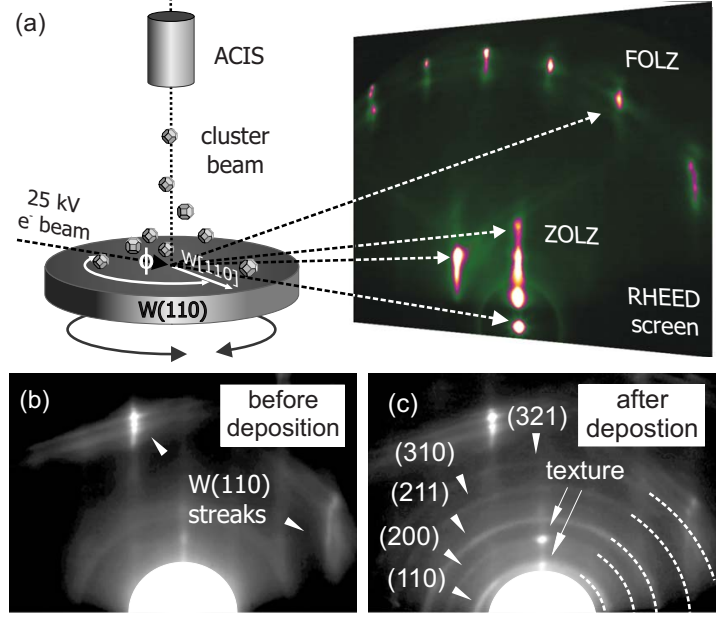

FIG. 1. (Color online) (a) Experimental setup for in situ electron-diffraction studies in a nanoparticle deposition experiment. The depicted diffraction pattern shows the zeroth (ZOLZ) and firstorder Laue zone (FOLZ) of the bare W(110) crystal surface. (b) Enlarged central section of a W(110) RHEED pattern before nanoparticle deposition, but at different orientation $\phi$ when compared to (a). (c) Diffraction pattern after deposition of Fe nanoparticles with $D=20 \mathrm{~nm}$

FWHM of $\Delta D / D \sim 0.15$. The kinetic energy of the clusters $E_{\text {kin }}<0.1 \mathrm{eV} /$ atom ensures soft-landing conditions. Prior to the cluster deposition the $\mathrm{W}(110)$ crystal is prepared by repeated cycles of heating in oxygen atmosphere and subsequent flashing up to $2400 \mathrm{~K}$ as described in the literature. ${ }^{22}$ The cleanliness and surface quality is checked by means of low-energy electron diffraction.

An enlarged central section of the RHEED pattern of $\mathrm{W}(110)$ at $\phi=69^{\circ}$ is given in Fig. 1(b). The deposition of a few tens of $\mathrm{Fe}$ nanoparticles per square micron with $D$ $=20 \pm 3 \mathrm{~nm}$ leads to the formation of several diffraction rings in addition to the unchanged $\mathrm{W}(110)$ features as shown in Fig. 1(c). These rings correspond to the (110), (200), (211), (310), and (321) Debye-Scherrer rings of bcc iron, and thus originate from randomly oriented nanoparticles. The (200) ring reveals an additional texture at the upper rim. This intense 200 spot stems from reflection by sets of (100) iron planes parallel to the sample surface and thus accounts for particles oriented with their (100) facets parallel to the substrate. A similar reflection is found on the (110) ring. This pattern is independent from the azimuthal sample orientation and therefore indicates particles resting with their (001) and (110) facets parallel to the substrate surface, but having a random azimuthal orientation. The same is found for particles with $D=25 \pm 3 \mathrm{~nm}$ and $13 \pm 2 \mathrm{~nm}$ as presented in Figs. 2(a) and 2(b)

Smaller particles show qualitatively different diffraction patterns, cf. Figs. 2(c) and 2(d) for $D=4.0 \pm 0.5 \mathrm{~nm}$ and $2.0 \pm 0.5 \mathrm{~nm}$, respectively. The reduced or vanishing ring intensities and the altered spot pattern hint at the onset of ordering. Rotating the samples reveals a periodic appearance and disappearance of certain reflexes as shown for the $4 \mathrm{~nm}$ particles in Figs. 3(a)-3(d). ${ }^{23}$ Analyzing the angulardependent data yields a $(110)_{\mathrm{Fe}} \|(110)_{\mathrm{W}}$ and $[001]_{\mathrm{Fe}} \|[001]_{\mathrm{W}}$
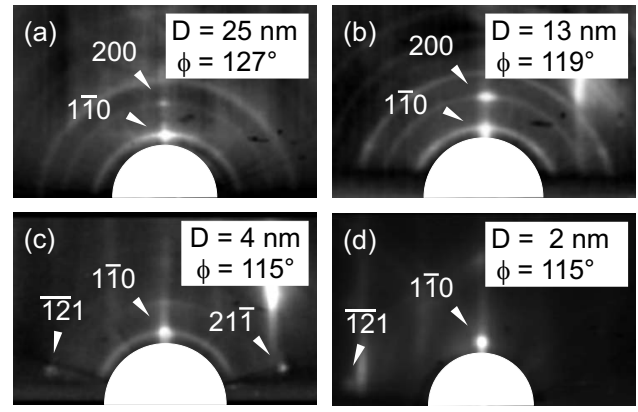

FIG. 2. Size-dependent Fe nanoparticle diffraction patterns. The larger particles in (a) and (b) give rise to a Debye-Scherrer powder pattern with a texture given by intense 200 and $1 \overline{1} 0$ reflexes (as denoted by arrows). Smaller particles show weak (c) or no (d) diffraction rings and an altered spot pattern, indicating the onset of spontaneous alignment relative to the substrate.

orientation, similarly to iron films epitaxially grown on W(110). Figure 3(e) shows a sketch of such an epitaxially aligned Fe particle with an ideal shape according to the Wulff theorem. (Real particles are slightly flattened upon deposition onto a substrate. ${ }^{16}$ ) Accordingly, the $4 \mathrm{~nm}$ particle sample consists of epitaxially ordered particles and a fraction of randomly oriented particles which gives rise to the remaining diffraction rings in Fig. 3. With $2 \mathrm{~nm}$ particles no rings appear, cf. Fig. 2(d), thus the full sample is epitaxially ordered. We may note that even for the smallest systems electron diffraction leads to distinct spots, which are not blurred or transformed into streaks. Correspondingly the clusters retain a three-dimensional shape and are not flattened to two-dimensional islands.

Our data are first discussed with respect to recent molecular-dynamics simulations. ${ }^{15}$ According to those, two possible alignment processes can be distinguished: (i) ther-
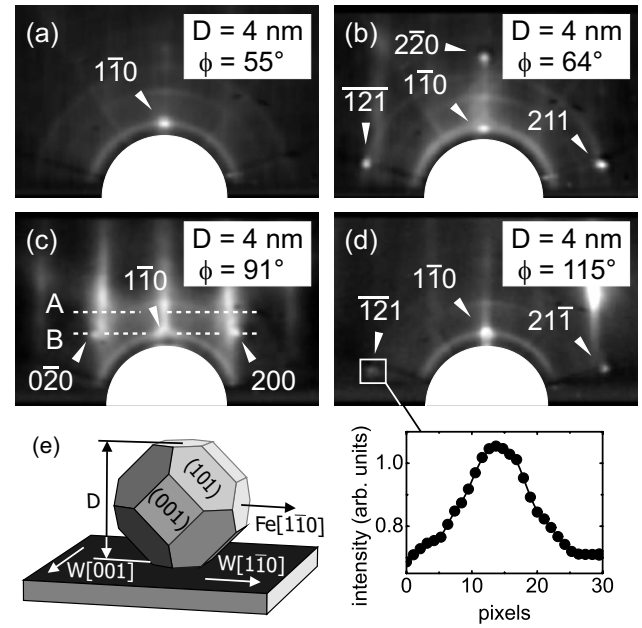

FIG. 3. Diffraction patterns recorded at different azimuthal orientations (a)-(d) reveal an epitaxial alignment of Fe nanoparticles with $D=4 \mathrm{~nm}$ on W(110). For clarity the inset in (d) shows a line scan through the faint $21 \overline{1}$ reflex. A sketch of a cluster (assuming a shape according to the Wulff theorem) in the corresponding $(110)_{\mathrm{Fe}} \|(110)_{\mathrm{W}}$ and $[001]_{\mathrm{Fe}} \|[001]_{\mathrm{W}}$ orientation on the substrate is shown in (e). 
mally activated motion of dislocations and (ii) mechanical melting. The latter requires that upon impact the cluster temporarily reaches the temperature for superheating which is usually somewhat higher than the respective thermal bulk melting temperature. ${ }^{15}$ To compare our data to these results we may estimate the temperature increase of the $2 \mathrm{~nm}$ particles from their kinetic energy $E_{\text {kin }}$ of the impact and the adhesion energy $E_{\text {adh }}$ released upon contact with the substrate. From the deposition parameters given above and the number of atoms being $N \sim 430$ we obtain $E_{\text {kin }} \leq 43 \mathrm{eV}$. The adhesion energy can in a first approximation be associated with the gain of surface energy given by $E_{\mathrm{adh}}=A\left(\gamma_{\mathrm{Fe}}+\gamma_{\mathrm{W}}\right)$ with $\gamma_{\mathrm{Fe}}=15 \mathrm{eV} / \mathrm{nm}^{2}$ and $\gamma_{\mathrm{W}}=25 \mathrm{eV} / \mathrm{nm}^{2}$ being the surface energies of iron and tungsten, respectively. ${ }^{24}$ The contact area of particles of this size, cf. Fig. 3(e), amounts to $A \sim 1 \mathrm{~nm}^{2}$ and thus leads to $E_{\text {adh }} \sim 40 \mathrm{eV}$. Taking into account that half of the energy is dissipated into the substrate the available energy in the nanoparticle amounts to $\Delta E$ $=\left(E_{\mathrm{adh}}+E_{\mathrm{kin}}\right) / 2 \sim 42 \mathrm{eV}$. Finally, applying the equipartition theorem expressed as $(3 / 2) \Delta T N k_{B}=\Delta E / 2$ (Ref. 15) yields a temperature increase of $\Delta T \sim 380 \mathrm{~K}$. Thus, a $2 \mathrm{~nm}$ particle may reach $T=300 \mathrm{~K}+\Delta T \sim 680 \mathrm{~K}$ in the present experiments, which is far below the melting temperature of bulk iron $T_{\mathrm{m}}=1808 \mathrm{~K}$ and still below the reduced melting temperature of $T_{\mathrm{m}}=900 \mathrm{~K}$ as expected for $2 \mathrm{~nm}$ particles. ${ }^{25}$ Similarly, for $4 \mathrm{~nm}$ particles one obtains a temperature increase of only $280 \mathrm{~K}$ while the reduced melting temperature is about $1680 \mathrm{~K}$. These estimates may therefore favor thermally activated motion of dislocations instead of melting as the relevant ordering mechanism. Further support for this conclusion comes from subsequent annealing experiments (not shown) where it is found that, e.g., the $13 \mathrm{~nm}$ particles realign (from random to epitaxial orientation) at about 640 $\mathrm{K}$, which is clearly below the melting temperature. However, besides activated motion of dislocations and melting of the whole particle other mechanisms should be considered. For instance, surface or local melting might be relevant, i.e., atoms closer to the substrate could form a transient liquid state at the time of impact. This could also contribute to the observed flattening of the particles. ${ }^{16}$

The properties of epitaxially ordered systems are strongly influenced by the symmetry and parameters of the involved crystal lattices. In the present case the different lattice parameters of iron $\left(a_{\mathrm{Fe}}=2.87 \AA\right)$ and tungsten $\left(a_{\mathrm{W}}=3.16 \AA\right)$ might induce noticeable strain in the aligned nanoparticles. Using the W(110) RHEED pattern as reference we can address this quantitatively in our data as shown in Fig. 4(a). This graph displays two line scans across the Fe $0 \overline{2} 0$ and the 200 spots and the related W streaks, respectively. Kinematical scattering theory shows that the horizontal separation of the Fe spots and the corresponding streaks of the W(110) surface lattice, $d_{\mathrm{Fe}}$ and $d_{\mathrm{W}(110)}$, respectively, is inversely proportional to the ratio of the corresponding lattice constants: $d_{\mathrm{W}(110)} / d_{\mathrm{Fe}}=a_{\mathrm{Fe}} / a_{\mathrm{W}}$. From the data of the 2 and $4 \mathrm{~nm}$ iron particles at different angles we find an (isotropic) ratio of about $d_{\mathrm{W}(110)} / d_{\mathrm{Fe}}=0.90 \pm 0.03$ as shown in Fig. 4(b). This reveals a bulklike ratio of the lattice constants within the experimental error bars, and thus surprisingly indicates a relaxed crystal lattice in the iron particles. However, from epi-

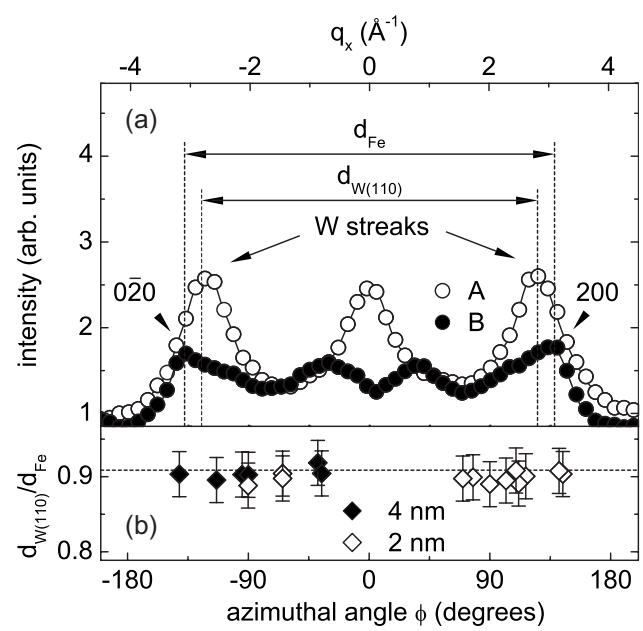

FIG. 4. (a) Intensity profiles along the dashed lines in Fig. 3(c). Open circles correspond to A and black circles to B. The peaks across the $0 \overline{2} 0$ and the $200 \mathrm{Fe}$ spots and the relevant W(110) streaks are denoted by the arrows. (b) The angular dependent ratio $d_{\mathrm{W}(110)} / d_{\mathrm{Fe}}$ as obtained from 2 and $4 \mathrm{~nm}$ particles.

taxially grown Fe films on $\mathrm{W}(110)$ it is known that the misfit-induced strain is relaxed after the first four atomic monolayers. ${ }^{26}$ An epitaxially ordered nanoparticle with $D$ $=2 \mathrm{~nm}$ [as shown in Fig. 3(e)] consists of a stack of about 11 atomic (110) layers. Similar to the films possible strain might thus be present only in the first few layers at the interface while the diffraction pattern is dominated by the larger number of relaxed layers.

In conclusion, using in situ reflection high-energy electron reflection we studied the alignment of mass-filtered iron nanocrystals upon deposition onto W(110). All investigated particles with diameters ranging from 2 to $25 \mathrm{~nm}$ showed a bulklike iron crystal structure and retain their threedimensional shape upon deposition. Particles with a diameter above about $4 \mathrm{~nm}$ tend to rest preferentially on their (001) and (110) surface facets, but with arbitrary in-plane orientation. Below that size the particles spontaneously align in a $(110)_{\mathrm{Fe}} \|(110)_{\mathrm{W}}$ and $[001]_{\mathrm{Fe}} \|[001]_{\mathrm{W}}$ configuration. Moreover, we find that misfit-induced strain in the epitaxially ordered particles might be restricted to the first few atomic layers at the interface. The data may further support recent molecular-dynamics simulations and give a hint at thermally activated dislocation motion as an effective ordering mechanism in low-energy deposition experiments. However, other effects as, e.g., transient melting close to the interface at the time of impact might be considered as well. Independent of the actual nature of the ordering mechanism, we expect that the observed size-dependent alignment is a general phenomenon and might be found in other systems. Thus, our findings may open an alternative route to magnetic nanostructures with tunable and uniform properties.

We gratefully acknowledge the fruitful discussion with J. Bansmann in the initial stage of this project. This work has been supported by the Deutsche Forschungsgemeinschaft (DFG) via the priority program SPP 1153 "Clusters in contact with surfaces" within the Contract No. KL 2188/1-3. 
*armin.kleibert@psi.ch

${ }^{1}$ A. P. Alivisatos, Science 271, 933 (1996).

${ }^{2}$ K.-H. Meiwes-Broer, Metal Clusters at Surfaces (Springer, Berlin, 2000).

${ }^{3}$ J. Bansmann, S. H. Baker, C. Binns, J. A. Blackman, J.-P. Bucher, J. Dorantes-Dávila, V. Dupuis, L. Favre, D. Kechrakos, A. Kleibert, K.-H. Meiwes-Broer, G. M. Pastor, A. Perez, O. Toulemonde, K. N. Trohidou, J. Tuaillon, and Y. Xie, Surf. Sci. Rep. 56, 189 (2005).

${ }^{4}$ H. Häkinen, S. Abbet, A. Sanchez, U. Heiz, and U. Landman, Angew. Chem. Int. Ed. 42, 1297 (2003).

${ }^{5}$ J. V. Barth, G. Costantini, and K. Kern, Nature (London) 437, 671 (2005).

${ }^{6}$ P. Jensen, Rev. Mod. Phys. 71, 1695 (1999).

${ }^{7}$ G. Renaud, R. Lazzari, C. Revenant, A. Barbier, M. Noblet, O. Ulrich, F. Leroy, J. Jupille, Y. Borensztein, C. R. Henry, J.-P. Deville, F. Scheurer, J. Mane-Mane, and O. Fruchart, Science 300, 1416 (2003).

${ }^{8}$ C. R. Henry, Prog. Surf. Sci. 80, 92 (2005).

${ }^{9}$ K. Bromann, C. Felix, H. Brune, W. Harbich, R. Monot, J. Buttet, and K. Kern, Science 274, 956 (1996).

${ }^{10}$ R. E. Palmer, S. Pratontep, and H.-G. Boyen, Nature Mater. 2, 443 (2003).

${ }^{11}$ H. Haberland, Z. Insepov, and M. Moseler, Phys. Rev. B 51, 11061 (1995).

${ }^{12}$ C. G. Zimmermann, K. Nordlund, M. Yeadon, J. M. Gibson, R. S. Averback, U. Herr, and K. Samwer, Phys. Rev. B 64, 085419 (2001).

${ }^{13}$ C. G. Zimmermann, M. Yeadon, K. Nordlund, J. M. Gibson, R. S. Averback, U. Herr, and K. Samwer, Phys. Rev. Lett. 83, 1163 (1999).
${ }^{14}$ B. Pauwels, G. Van Tendeloo, W. Bouwen, L. T. Kuhn, P. Lievens, H. Lei, and M. Hou, Phys. Rev. B 62, 10383 (2000).

${ }^{15}$ T. T. Järvi, A. Kuronen, K. Meinander, K. Nordlund, and K. Albe, Phys. Rev. B 75, 115422 (2007).

${ }^{16}$ A. Kleibert, F. Bulut, R. K. Gebhardt, W. Rosellen, D. Sudfeld, J. Passig, J. Bansmann, K.-H. Meiwes-Broer, and M. Getzlaff, J. Phys.: Condens. Matter 20, 445005 (2008).

${ }^{17}$ O. Fruchart, P. O. Jubert, M. Eleoui, F. Cheynis, B. Borca, P. David, V. Santonacci, A. Lienard, M. Hasegawa, and C. Meyer, J. Phys.: Condens. Matter 19, 053001 (2007).

${ }^{18}$ A. Kleibert, K.-H. Meiwes-Broer, and J. Bansmann, Phys. Rev. B 79, 125423 (2009).

${ }^{19}$ R.-P. Methling, V. Senz, E.-D. Klinkenberg, T. Diederich, J. Tiggesbäumker, G. Holzhüter, J. Bansmann, and K.-H. MeiwesBroer, Eur. Phys. J. D 16, 173 (2001).

${ }^{20}$ A. Kleibert, J. Passig, K.-H. Meiwes-Broer, M. Getzlaff, and J. Bansmann, J. Appl. Phys. 101, 114318 (2007).

${ }^{21}$ J. Passig, K. Meiwes-Broer, and J. Tiggesbäumker, Rev. Sci. Instrum. 77, 093304 (2006).

${ }^{22}$ M. Bode, R. Pascal, and R. Wiesendanger, Surf. Sci. 344, 185 (1995).

${ }^{23}$ See supplementary material at http://link.aps.org/supplemental/ 10.1103/PhysRevB.81.073412 for a movie showing the evolution of the diffraction pattern when rotating the sample azimuthally.

${ }^{24}$ L. Vitos, A. V. Ruban, H. L. Skriver, and J. Kollár, Surf. Sci. 411, 186 (1998).

${ }^{25}$ K. K. Nanda, S. N. Sahu, and S. N. Behera, Phys. Rev. A 66, 013208 (2002).

${ }^{26}$ H. Bethge, D. Heuer, Ch. Jensen, K. Reshöft, and U. Köhler, Surf. Sci. 331-333, 878 (1995). 